\title{
Deutschland soll ein international sichtbarer Innovations- standort in der Gesundheitsforschung werden
}

Die Innere Medizin wird derzeit von vielen Neuerungen und echten Innovationssprüngen bereichert. Diese betreffen zum Beispiel die Behandlung der Hepatitis-C-Virus(HCV)-Infektion, die durch neue Wirkstoffkombinationen fast immer in Remission gebracht werden kann. Eines der sich am schnellsten entwickelnden Gebiete ist vermutlich die Onkologie. Kaum ein Monat vergeht, in dem nicht über ein neues, viel versprechendes Medikament in einer der führenden Zeitschriften der Medizin berichtet wird.

Immer mehr kommt es zur Pathogenese-orientierten Behandlung von Krebs. Krebs entsteht auf dem Boden genetischer Aberrationen in der transformierenden Zelle, die zunehmend entschlüsselt werden. Daher werden immer öfter vor Einleitung von Therapien aufwändige diagnostische Verfahren nötig - sie zu strukturieren ist eine komplexe und große organisatorische Aufgabe. Deshalb ist in der Onkologie eine Entwicklung zu beobachten, welche die Behandlung von Tumorpatienten zunehmend in Zentren oder Netzwerke aus Krankenhäusern und Ärzten verlegt. Die dafür nötigen Strukturen wurden im Nationalen Krebsplan sehr gut festgehalten.

Weniger erfreulich ist, dass immer noch viel zu wenige der therapeutischen Innovationen primär in Europa oder Deutschland entwickelt werden. Es ist daher nötig, den Wissenschaftsstandort Deutschland mit Bezug auf die Lebenswissenschaften und die Gesundheitsforschung neu zu definieren. Hierfür sind folgende Schritte wichtig: 1. Änderung der Perspektive:

a. Die Gesundheitswirtschaft ist einer der größten Wirtschaftszweige und Arbeitgeber unseres Landes und sollte positiv gesehen werden.

b. Fortschritt für die Patienten durch verbesserte Therapien oder Diagnoseverfahren ist eine positive Errungenschaft und darf nicht nur als Kostenfaktor betrachtet werden.

2. Verbesserung der Strukturen und Förderung:

a. Durch verbesserte Strukturen und Förderung der experimentellen wie klinischen Forschung in der Industrie, aber vor allem an den Universitäten muss Deutschland wieder zurückfinden zur Tradition des führenden Innovationsstandortes für die Entwicklung neuer Arzneien.

b. Die extremen bürokratischen Auflagen für die klinische Forschung müssen reduziert werden (selbstverständlich unter Beachtung der Patientensicherheit).

Hinzu kommt, dass die Genehmigungs- und Zulassungsverfahren von Medikamenten immer länger dauern und immer höhere Auflagen erfüllen müssen. Auch dies behindert den Fortschritt und erhöht die Arzneimittelpreise. In Gebieten mit raschem Erkenntnisfortschritt können die derzeit erforderlichen, zahlreichen Prüf- und Zulassungsverfahren kaum mehr Schritt halten mit dem Tempo der Innovationen. Dies ist besonders zu beobachten in der Onkologie. Die Neueinführung von neuen Medikamenten wird dadurch häufig stark behindert und verzögert. Außerdem begünstigt es die Entstehung von Monopolen, weil nur noch große Firmen die Entwicklung neuer Substanzen betreiben können. Dies alles ist nicht im Sinne der Patienten. Was ist hier zu tun? 1. Schnelle Zulassung, schnelle Erstattung von echten Innovationen. Dies ist eine Pflicht der Behörden gegenüber dem Bürger.

2. Um die Erkenntnisse über den Wert neuer Substanzen oder Therapieprinzipien nach der $\mathrm{Zu}-$ lassung zu verbessern, sollte die dokumentierte Behandlung in Studien zur Regel werden.

3. Diese Studien sollten von der Wissenschaft konzipiert („investigator-initiated trials“) und unabhängig von den Interessen der Industrie oder der Krankenkassen, beispielsweise aus einem Pool, finanziert werden.

Diese Maßnahme könnte dazu führen, dass unser Land sehr schnell einer der weltweit führenden Innovationsstandorte im Gebiet der Gesundheitswissenschaften und -wirtschaft würde. Deutschland könnte eine Art „Silicon Valley“ der Gesundheitsforschung werden. Durch seine hohe Wirtschaftskraft und die Existenz vernetzter Strukturen (z.B. Kompetenznetze der Medizin; Gesundheitsforschungszentren), die exzellente Qualität der Krankenversorgung, die kurzen Distanzen zwischen unterschiedlichen Standorten und auch die primär solidarische Ausrichtung des Gesundheitswesens sind dafür ideale Voraussetzungen geschaffen, die in vielen Regionen der Welt so nicht gegeben sind.

Auf den kommenden großen Kongressen, dem Deutschen Krebskongress 2014, vom 19.-22. Februar 2014 in Berlin, und dem Kongress der Deutschen Gesellschaft für Innere Medizin, vom 26.-29. April 2014 in Wiesbaden, wird sicher über diese wichtigen Themen diskutiert werden. $\mathrm{Zu}$ beiden Kongressen lade ich Sie ebenso herzlich ein wie zur Lektüre dieses Heftes.
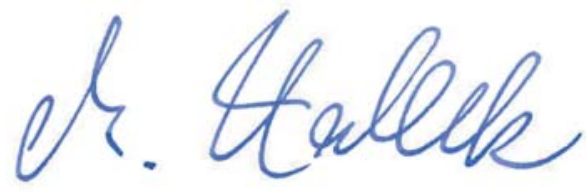

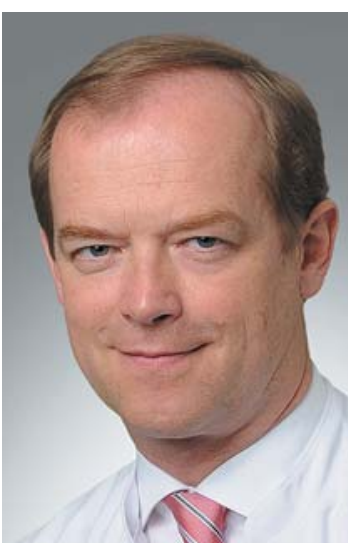

M. Hallek

Institut

Klinik I für Innere Medizin,

Uniklinik Köln

Bibliografie

Dol 10.1055/s-0033-1360090

Dtsch Med Wochenschr 2014;

139: 227 . (c) Georg Thieme Verlag KG · Stuttgart · New York . ISSN 0012-0472

Korrespondenz

Prof. Dr. Michael Hallek

Klinik I für Innere Medizin, Uniklinik Köln Kerpener Str. 62 50937 Köln 